\title{
Utilização da Minilaparoscopia em Pacientes com Algia Pélvica
}

\author{
Office Microlaparoscopy in Patients with Chronic Pelvic Pain
}

Waldir Pereira Modotte, Rogério Dias

\begin{abstract}
RESUMO
Objetivo: analisar o uso da vídeo-minilaparoscopia no diagnóstico das causas de algia pélvica, bem como sua aplicabilidade.

Métodos: foram estudadas prospectivamente 32 pacientes com algia pélvica, com idade média de 30 anos, submetidas a vídeo-minilaparoscopia. Analisamos nesse trabalho: a duração do procedimento, o tempo de permanência no centro de recuperação, a qualidade técnica da imagem, os achados laparoscópicos, a tolerância do método anestésico, sob sedação consciente, a morbidade pós-operatória e a aceitação do procedimento cirúrgico pela paciente. Resultados: o tempo médio de duração foi de 19 minutos, o tempo médio de permanência no centro de recuperação de 43 minutos e a qualidade de imagem excelente ou boa em todas as pacientes selecionadas. Foram encontrados os seguintes achados laparoscópicos: 34,4\% de endometriose, 28,1\% de aderências pélvicas, 12,5\% de varizes pélvicas e 25\% normais. Baseado no critérios de Bordhal et al. ${ }^{1}$, foi observada uma baixa freqüência de manifestação dolorosa durante a anestesia local $(12,5 \%)$ e relativo desconforto $(46,9 \%)$ na realização do pneumoperitônio. Observou-se que o método apresenta tolerância muito boa e boa em 96,9\%, utilizando-se os critérios de Milki e Tazuke ${ }^{2}$. A morbidade do método, 24 horas após o procedimento, segundo os critérios de Chung et al. ${ }^{3}$, mostrou elevada freqüência de dor no local da incisão $(59,4 \%)$ e sonolência (43,8\%). Apenas 3,1\% referiu dor durante o procedimento, mostrando boa aceitação do método.

Conclusões: a tolerância ao método anestésico, a baixa morbidade pós-operatória e a aceitação do procedimento cirúrgico pelas pacientes fazem da vídeo-minilaparoscopia método propedêutico extremamente importante nas pacientes portadoras de algia pélvica.
\end{abstract}

PALAVRAS-CHAVE: Algia pélvica. Cirurgia ambulatorial. Endometriose. Laparoscopia.

\section{Introdução}

Algia pélvica (AP) é definida como dor na região pélvica, acompanhada de distúrbios sociais e/ ou psicológicos ${ }^{4}$. Por outro lado, Milburn et al. ${ }^{5}$ amplia essa definição como dor abdominal ou pélvica, não-cíclica, com duração mínima de seis meses.

Algia pélvica é uma queixa muito freqüente nos consultórios ginecológicos. Afeta cerca de 12-33\% de todas as mulheres no período reprodutivo, porém é ainda um enigma para os clínicos pela dificuldade de se estabelecer a etiologia ${ }^{6}$. O diagnós-

Departamento de Ginecologia e Obstetrícia da Faculdade de Medicina de Botucatu - UNESP.

Correspondência:

Waldir Pereira Modotte

Rua Nágila Jubran, 40 - Jd Europa

19800-000 - Assis - SP

e-mail: iamulher@femanet.com.br tico clínico é dificil, porque poucas vezes se encontram anormalidades orgânicas. Pacientes com algia pélvica são ansiosas e apresentam depressão associada, aumentando ainda mais a complexidade dessa sindrome. Apresentam graves rupturas na situação ocupacional, marital e social, com conseqüente impacto na qualidade de vida. São pacientes que respondem mal aos diversos esquemas terapêuticos em ginecologia e, não raramente, são submetidas a sucessivas abordagens cirúrgicas, sem sucesso ${ }^{7,8}$.

Com freqüencia, essas pacientes recebem o diagnóstico de doença inflamatória pélvica, sendo tratadas com sucessivos esquemas de antibióticos, analgésicos e antiinflamatórios, e mesmo assim, apresentam recorrência da sintomatologia ${ }^{9}$. O diagnóstico diferencial é complexo, o que, na maioria das vezes, obscurece o caminho do clínico na busca da etiologia. 
No passado, pacientes portadoras de algia pélvica, quando não respondiam ao tratamento clínico, eram submetidas à laparotomia na tentativa de elucidar a causa da sua morbidade ${ }^{10}$. Atualmente a algia pélvica é responsável por $15-40 \%$ das indicações para laparoscopias diagnósticas e $12 \%$ das indicações para histerectomias nos Estados Unidos ${ }^{11,12}$.

$\mathrm{Na}$ prática ginecológica moderna, a vídeolaparoscopia é opção de grande valor na abordagem desse sintoma, quando não há o diagnóstico clínico 9 . Inúmeros autores recomendam a inclusão da laparoscopia no protocolo da investigação da dor pélvica, pelas vantagens que ela representa nesse contexto ${ }^{7,9,11}$.

A vídeo-laparoscopia, possibilitando visão direta da cavidade pélvica e abdominal, é útil para estabelecer o diagnóstico definitivo ou, pelo menos, para excluir a presença de patologias pélvicas. Baseado nos achados laparoscópicos, o clínico tem a orientação para o tratamento adequado, porém, a grande contribuição do diagnóstico laparoscópico é o fato de se evitarem laparotomias, que é tratamento de elevada morbidade e alguma mortalidade ${ }^{13,14}$.

A laparoscopia convencional, com ópticas de 5 ou $10 \mathrm{~mm}$ de diâmetro, pode ser realizada sob anestesia local, com laparoscópios de "lentes redondas". Entretanto, mesmo em excelentes condições, é procedimento associado a moderado desconforto. A utilização de laparoscópios de menor diâmetro (2-3 mm), chamados de minilaparoscópios, está disponível e representa alternativa aos laparoscópios convencionais, pelo desconforto operatório ainda menor, principalmente para os procedimentos diagnósticos ${ }^{5}$.

Em 1996, Milki e Tazuke ${ }^{2}$, numa série de 175 procedimentos mini-laparoscópicos, com a finalidade de transferência intratubária de gametas (GIFT), concluíram que a realização da vídeominilaparoscopia sob anestesia local é efetiva, é bem aceita pelos cirurgiões e é preferida pelas pacientes. Seu estudo mostrou redução de custo, maior flexibilidade de agendamento e alta taxa de sucesso.

A realização da vídeo-minilaparoscopia sob anestesia local é um dos mais recentes avanços da cirurgia minimamente invasiva e representa a interseção de duas tecnologias. A primeira, o uso do laparoscópio de menor calibre $(2 \mathrm{~mm})$ e instrumentação acessória, de calibre também reduzido $(2-3 \mathrm{~mm})$. A segunda, a técnica da realização da laparoscopia sob anestesia local, podendo ser realizada no ambiente hospitalar, de modo ambulatorial, ou até mesmo em consultório médico convencional, equipado de monitores de segurança ${ }^{15}$.

A vídeo-minilaparoscopia, além de importan- te no diagnóstico, contribui na execução de alguns procedimentos laparoscópicos de pequeno porte, tais como: cromotubagem, lise de aderências finas, biópsia e eletrocauterização de lesões endometrióticas, laqueadura tubárea, aspiração de líquido intraperitoneal, GIFT e realização do mapeamento de dor ${ }^{16}$.

O objetivo deste trabalho foi o de analisar o uso da vídeo-minilaparoscopia no diagnóstico das causas de algia pélvica, bem como sua aplicabilidade.

\section{Pacientes e Métodos}

Trata-se de um estudo prospectivo que incluiu 32 mulheres portadoras de algia pélvica, atendidas na Clínica do Instituto de Atendimento à Mulher, na cidade de Assis, estado de São Paulo, no período compreendido entre janeiro e dezembro de 1998. Analisamos nesse trabalho: a duração do procedimento, o tempo de permanência no centro de recuperação, a qualidade técnica da imagem, os achados laparoscópicos, a tolerância ao método anestésico, sob sedação consciente, a morbidade pós-operatória e a aceitação do procedimento cirúrgico pela paciente.

Foram selecionadas 62 pacientes com algia pélvica, candidatas à laparoscopia diagnóstica. Destas, foram excluídas 30 pacientes, pelos seguintes motivos: diagnóstico clínico que justificava a dor pélvica, índice de massa corpórea maior que $30 \mathrm{~kg} /$ $\mathrm{m}^{2}$ (peso em $\mathrm{kg}$ dividido pelo quadrado da altura em $\mathrm{m})$, mais de três cirurgias pélvicas anteriores e contra-indicações convencionais para a laparoscopia, como por exemplo: doença cardiopulmonar preexistente e doença neurológica. Também foram excluídas as pacientes que apresentaram história pregressa de processo alérgico ou história prévia de complicações atribuídas à anestesia e alteração nos exames pré-operatório ${ }^{8}$ : hemograma, glicemia de jejum, coagulograma, uréia, creatinina, urina rotina e urocultura.

As 32 pacientes selecionadas foram submetidas à vídeo-minilaparoscopia diagnóstica, com técnicas de anestesia local e sedação consciente, utilizando equipamentos adequados, realizado em ambiente hospitalar, de modo ambulatorial.

Esse projeto de pesquisa segue os critérios exigidos pela Comissão de Ética e Pesquisa da Faculdade de Medicina de Botucatu - UNESP.

Empregamos a sedação consciente, definida como depressão minima da consciência, com preservação da função respiratória, produzida por métodos farmacológicos e/ou não-farmacológicos, mantendo a capacidade de responder à estimulação 
fisica apropriada ou comando verbal ${ }^{3}$.

As pacientes foram levadas ao centro cirúrgico após receber medicação endovenosa de benzodiazepínico (dosagem de $10 \mathrm{mg}$, diluídos em $10 \mathrm{ml}$ de água destilada). Com o intuito de sedação consciente utilizou-se midazolam endovenoso, na dosagem de $10 \mathrm{mg}$ e fentanila, na dosagem de $0,05 \mathrm{mg} / \mathrm{kg}$ de peso. Quando necessário, dose adicional desta medicação foi utilizada.

Foi realizada anestesia local, utilizando-se $10 \mathrm{ml}$ de lidocaína (Xilocaína ${ }^{\circledR}$ ) a $1 \%$, no bloqueio paracervical, sendo 2,5 ml em cada quadrante, 8$12 \mathrm{ml}$ de bupivacaína (Marcaina ${ }^{\circledR}$ ) a $0,25 \%$, na anestesia da região umbilical, e 5-10 ml na região suprapúbica. Foi realizada uma pequena incisão intra-umbilical, de $3 \mathrm{~mm}$ de extensão, com bisturi de lâmina número 11 , e outra semelhante na região suprapúbica.

O pneumoperitônio foi realizado sempre de modo cuidadoso, mantendo pressão intra-abdominal inferior a $10 \mathrm{mmHg}$, com a utilização de insuflador eletrônico. Instalado o pneumoperitônio, introduziu-se o complexo trocar (agulha e camisa) e óptica da vídeo-minilaparoscopia, de marca Storz (Tuttlingen, Alemanha). Um trocar acessório de $3 \mathrm{~mm}$ foi introduzido na região supra-umbilical, seguido da utilização de um palpador (Storz Tuttlingen, Alemanha).

Após a instalação de todo o equipamento, iniciou-se a exploração vídeo-laparoscópica, que consiste na inspeção sistemática do abdome superior, abdome inferior e cavidade pélvica, seguido do diagnóstico laparoscópico propriamente dito.

Para liberação das pacientes da recuperação, obedeceu-se aos critérios de sistema de escore de liberação (PADSS - Post-anesthetic Discharge Scoring System) ${ }^{3}$, realizado cada 5 minutos.

Avaliou-se a tolerância ao método, observando o desconforto em determinados momentos do procedimento, conforme os critérios de Bordahl et al. ${ }^{3}$. Ainda no momento da liberação das pacientes da recuperação, com a mesma finalidade de avaliar a tolerabilidade do método, uma avaliação foi aplicada seguindo os critérios de Milki e Tazuke ${ }^{2}$, conforme segue:

Muito Boa - mínimo ou nenhum desconforto, nenhuma ansiedade e medicação adicional desnecessária.

Boa - alguma dor ou ansiedade e medicação adicional desnecessária.

Aceitável - desconforto e ansiedade várias vezes durante o procedimento, necessidade de medicação adicional, com boa melhora da sintomatologia dolorosa.

Ruim - desconforto e/ou ansiedade freqüentes, necessidade de medicação adicional, sem melho- ra da sintomatologia dolorosa.

Péssima ou Proibitiva - necessidade de interrupção do procedimento, devido ao intenso desconforto e/ou ansiedade, não-controlados com medicação adicional.

Com o objetivo de avaliar a morbidade do procedimento, as pacientes foram interrogadas 24 horas após a realização do exame, conforme questionário elaborado por Chung et al. ${ }^{3}$.

Sete dias após o procedimento cirúrgico, as pacientes foram novamente interrogadas quanto a dor ou qualquer desconforto durante o procedimento laparoscópico.

As variáveis quantitativas foram analisadas descritivamente, com determinação de medidas de tendência central (médias e medianas) e de variabilidade (desvio padrão e coeficiente de variação). Foi realizada uma determinação de freqüência de ocorrência para as variáveis classificatórias, utilizando-se o cálculo das freqüências absolutas e relativas ${ }^{17}$.

\section{Resultados}

O tempo médio de duração do procedimento foi de 19 minutos (mínimo de 10 e máximo de 40 minutos), com mediana de 15 minutos (Tabela 1). O tempo médio de permanência no centro de recuperação foi de 43 minutos (mínimo de $20 \mathrm{~min}$ e máximo de 95 minutos), com mediana de $40 \mathrm{mi}-$ nutos. A qualidade técnica da imagem foi excelente ou boa em todos os procedimentos realizados $(81,3 \%$ e $18,7 \%$, respectivamente).

Tabela 1 - Distribuição das 32 pacientes com algia pélvica, em relação à duração do procedimento da vídeo-minilaparoscopia. Estatísticas calculadas.

\begin{tabular}{lrr}
\hline Duração (minutos) & $\mathbf{n}$ & $\%$ \\
\hline 10 & 5 & 15,6 \\
15 & 13 & 40,6 \\
20 & 6 & 18,8 \\
25 & 2 & 6,3 \\
30 & 3 & 9,4 \\
35 & 1 & 3,1 \\
40 & 2 & 6,3 \\
Total & 32 & 100 \\
\hline Média = 19 minutos; mediana = 15 minutos; coeficiente de variação $=70,5 ;$ desvio padrão \\
$=8,40$.
\end{tabular}

Foram encontradas aderências pélvicas em $28,1 \%$ das pacientes examinadas, endometriose em $34,4 \%$ e $12,5 \%$ apresentavam varizes pélvicas. Em $25 \%$ das pacientes o exame foi normal (Figura 1). 


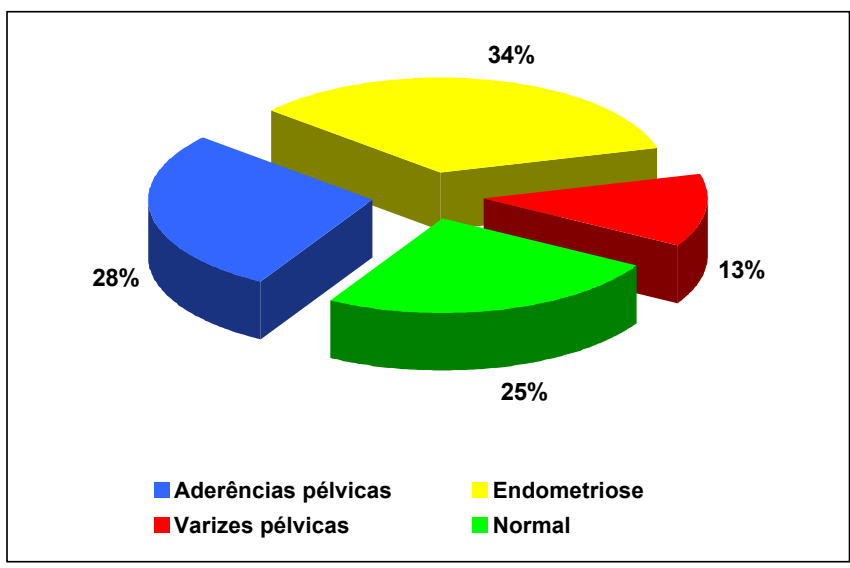

Figura 1 - Diagnósticos obtidos pela vídeo-minilaparoscopia em 32 pacientes com algia pélvica.

Utilizando-se os critérios de Bordahl et al. ${ }^{1}$, encontramos desconforto leve em apenas 12,5\% das pacientes submetidas ao método durante a anestesia local e desconforto leve e moderado em $50 \%$ das pacientes, que se manteve por pequeno período. Apenas 3,1\% das pacientes referiram desconforto durante a permanência no centro de recuperação.

Utilizando-se os critérios de Milki e Tazuke ${ }^{2}$, houve tolerância muito boa ou boa em $96,9 \%$ das pacientes submetidas ao método. Apenas 3,1\% das pacientes referiram tolerância aceitável.

$\mathrm{Na}$ avaliação da morbidade do método, 24 horas após o procedimento, obtivemos $59,4 \%$ de queixa de dor na incisão e $43,8 \%$ de sonolência (Tabela 2).

Tabela 2 - Distribuição das respostas para avaliação da morbidade da vídeominilaparoscopia, em pacientes com algia pélvica, obtidas 24 horas após o procedimento.

\begin{tabular}{lcc}
\hline Morbidade & \multicolumn{2}{c}{ Resposta afirmativa } \\
& $\mathbf{n}$ & $\%$ \\
\hline Dor no local & 19 & 59,4 \\
Dor em outro local & 9 & 28,1 \\
Febre & 1 & 3,1 \\
Utilização de analgésico & 16 & 50 \\
Náuseas/vômitos & 2 & 6,3 \\
Cefaléia & 1 & 3,1 \\
Sonolência & 14 & 43,8 \\
Tontura/vertigem & 5 & 15,6 \\
Fraqueza/desmaio & 2 & 6,3 \\
\hline
\end{tabular}

Das 32 pacientes, 31 (96,9\%) responderam que não sentiram nenhum tipo de dor durante o procedimento. Apenas uma $(3,1 \%)$ respondeu que apresentou dor durante o procedimento e referiu escore moderado.

\section{Discussão}

A algia pélvica é um sintoma que afeta a mulher primariamente no periodo reprodutivo, porém, encontram-se com relativa freqüência pacientes com dor pélvica fora desse período ${ }^{18}$. A média de idade das nossas pacientes foi 30 anos, variando de um mínimo de 16 anos a um máximo de 41 anos. A literatura é diversificada em relação a idade média de ocorrência desse sintoma ${ }^{5,11}$.

O tempo de duração da vídeo-minilaparoscopia mostra que o procedimento é de curta duração. O tempo de permanência no centro de recuperação também é curto. Esses achados estão de acordo com a literatura e confirmam a possibilidade de utilizar o método como parte de um protocolo na rotina da pesquisa da algia pélvica ${ }^{9,14,15,19-21}$.

Feste $^{22}$, numa série de 36 mulheres, concluiu que a vídeo-minilaparoscopia proporciona visão clara da cavidade pélvica, sem a necessidade de anestesia geral. A qualidade da imagem obtida foi suficiente para um diagnóstico eficaz, reforçando os achados de literatura ${ }^{15}$.

Os achados laparoscópicos são coincidentes com a literatura, onde $25 \%$ das pacientes não apresentaram anormalidades à luz da laparoscopia. Os diagnósticos positivos estão distribuídos principalmente entre endometriose, aderências pélvicas e varizes pélvicas.

Quanto à tolerância ao método anestésico, sob sedação consciente, observou-se que o método é bem tolerado. A ocorrência de desconforto leve durante a anestesia local foi baixa. O desconforto sentido pela maioria das pacientes durante o pneumoperitônio também foi baixo, sendo mantido por pouco tempo, principalmente no início da insuflação. Pode se inferir que as pacientes apresentaram boa tolerância com relação ao método. Milki e Tazuke ${ }^{2}$, analisando 175 procedimentos laparoscópicos sob anestesia local, encontraram tolerância muito boa ou boa em $89 \%$ das pacientes, com infertilidade submetidas ao método, para procedimentos de GIFT.

$\mathrm{Na}$ avaliação da morbidade do método, foi observado elevada freqüência de dor no local da incisão e sonolência, levando a maior necessidade de analgésicos. Em relação aos demais sintomas, esses foram encontrados em baixa freqüência. Esses achados mostram que o método apresenta baixa morbidade.

$\mathrm{Na}$ avaliação da aceitabilidade do método apenas $3,1 \%$ das pacientes referiu dor durante o procedimento. Isso se deve à ministração de medicação sedativa, aplicada antes do procedimento, que apresenta um efeito amnésico.

O presente estudo tem a pretensão de suge- 
rir o método como rotina de um protocolo de investigação da algia pélvica. Para uma utilização mais liberal da laparoscopia na investigação da dor pélvica escolhemos realizá-la pela vídeominilaparoscopia, sob anestesia local e sedação consciente. As vantagens são bastante conhecidas: diminuição no tempo de espera para agendamento, no tempo operatório, no tempo de recuperação anestésica, menor custo do pré-operatório, intra e pós-operatório e menor necessidade de permanência hospitalar, de medicamentos e de curativos ${ }^{2,13,14,16,18,19,22}$.

A vídeo-minilaparoscopia oferece ao ginecologista a opção de abordagem laparoscópica com qualidade de imagem, a segurança na sua realização, a invasão mínima e a oportunidade de realizála com anestesia local em ambiente não hospitalar. O procedimento mostrou ser de curta duração, com baixo tempo de permanência na sala de recuperação e de boa qualidade técnica de imagem.

\section{SUMMARY}

Purpose: to study the usefulness of minilaparoscopy in diagnosing the cause of pelvic pain.

Methods: women with pelvic pain were prospectively analyzed and underwent an office video-microlaparoscopy. We analyzed the data regarding procedure time, stay in the recovery room, acceptance of anesthesia, and morbidity. Results: the average procedure time of the office videomicrolaparoscopy was $19 \mathrm{~min}$, the average stay for recovery was $43 \mathrm{~min}$, and the technical quality of the image was excellent or good in $100 \%$ of the selected patients. The following laparoscopic findings were reported: $34.4 \%$ endometriosis, $28.1 \%$ pelvic adhesion, $12.5 \%$ pelvic varices, and 25\% normal. Based on Bordhal et al. 's ${ }^{1}$ criteria, a low frequency of pain manifestation during local anesthesia (12.5\%) and discomfort on pneumoperitoneum (46.9\%) were noticed. It could also be observed that, according to Milki and Tazuke's ${ }^{2}$ criteria, the tolerance to the method was excellent and good (96.9\%). Twentyfour hours after the procedure the morbidity rate was in accordance with Chung et al. s $^{3}$ criteria, showing a high frequency of pain at the incision area (59.4\%) and sleepiness $(43.8 \%)$. Only $3.1 \%$ reported they felt pain during the procedure, which shows the acceptance of the method by the patients.

Conclusions: the acceptance of anesthesia and of the surgical procedure and the low morbidity allow the use of minilaparoscopy as a very important method in investigating patients with pelvic pain.

KEY WORDS: Chronic pelvic pain. Office laparoscopy. Office surgery. Laparoscopy.

\section{Referências}

1. Bordahl PE, Raeder JC, Nordentoft J, Kirste U, Refsdal A. Laparoscopic sterilization under local or general anesthesia? A randomized study. Obstet Gynecol 1993; 81:137-41.

2. Milki AA, Tazuke SI. Office laparoscopy under local anesthesia for gamete intra-fallopian transfer: technique and tolerance. Fertil Steril 1997; 68:128-32.

3. Chung F, Un V, Su J. Postoperative symptoms 24 hours after ambulatory anaesthesia. Can J Anaesth 1996; 43:1121-7.

4. Howard, FM. The role of laparoscopy in chronic pelvic pain: promise and pitfalls. Obstet Gynecol Surv 1993; 48:357-87.

5. Milburn A, Reiter RC, Rhomberg A. Multidisciplinary approach to chronic pelvic pain. Obstet Gynecol Clin North Am 1993; 20:643-61.

6. Steege JF, Metzger DA, Levy B. Scope of the problem in chronic pelvic pain: an integrated approach. In - (editors). Chronic pelvic pain. 2nd ed. Philadelphia: Saunders; 1998.p.1-4.

7. Carter JE. Diagnosis and treatment of the causes of chronic pelvic pain. J Am Ass Gynecol Laparosc 1996; 3 Suppl: S5-6.

8. Rapkin AJ. Pelvic pain and dysmenorrhea. In: Berek JS, editor. Novak's gynecology. $12^{\text {th }}$ ed. London: Williams \& Wilkins; 1996. p. 399-428.

9. al-Suleiman SA. Laparoscopy in the management of women with chronic pelvic pain. Aust N Z J Obstet Gynecol 1991; 31:63-5.

10.Slocumb JC. Operative management of chronic abdominal pelvic pain. Clin Obstet Gynecol 1990; 33:196-204.

11.Howard FM. Laparoscopic evaluation and treatment of women with chronic pelvic pain. J Am Ass Gynecol Laparosc 1994; 1:325-31.

12.Wilcox LS, Koonin LM, Pokras R, Strauss LT, Xia Z, Peterson HB. Hysterotomy in the United States, 1988-90. Obstet Gynecol 1994; 83:549-55.

13.Lundberg WI, Wall JE, Mathers JE. Laparoscopy in the evaluation of pelvic pain. Obstet Gynecol 1973; 42:872-6.

14. Reiter RC, Milburn A. Management of chronic pelvic pain. Postgrad Obstet Gynecol 1992; 12:114-20.

15.Palter SF, Olive DL. Office micro-laparoscopy under local anesthesia for chronic pelvic pain. J Am Ass Gynecol Laparosc 1996; 3:359-64.

16.Sanfelippo J. Micro-laparoscopy under local: chronic pelvic pain. Syllabus of the $45^{\text {th }}$ Annual Meeting of the American College of Obstetricians and Gynecologists; 1997 Apr 29-30; Las Vegas, USA. p.1-8. 
17. Curi PR. Metodologia e análise da pesquisa em ciências biológicas. $2^{a}$ ed. Botucatu: Tipomic, 1998.

18.Steege JF. Pain mapping procedures. Syllabus of the $27^{\text {th }}$ Annual Meeting of the AAGL; 1998 Nov 11; Atlanta, USA. p. 43-60.

19.Palter SF. Office micro-laparoscopy under local anesthesia. Syllabus of the $45^{\text {th }}$ Annual Clinical Meeting of the American College of Obstetricians and Gynecologists; 1997 Apr 29-30; Las Vegas, USA. p.32-56.
20.Mehta PV. A total of 250136 laparoscopic sterilizations by a single operator. Br J Obstet Gynaecol 1989; 96:1024-34.

21.Kontoravdis A, Chryssilkopoulos A, Hassiakos D, Liapis A, Zourlas PA. The diagnostic value of laparoscopy in 2365 patients with acute and chronic pelvic pain. Int J Gynaecol Obstet 1996; 52:243-8.

22.Feste JR. Use of optical catheter for diagnostic office laparoscopy. J Reprod Med 1996; 41:307-12. 